\title{
HPLC Determination of 6-Methylcoumarin and 3-Pyridine Methanol in Toiletries for Oral Hygiene
}

\author{
Daniela De OrSI,* Luigi Gagliardi,* Adriana Bolasco,* and Domenica Tonelli**† \\ * Laboratorio di Chimica del Farmaco, Istituto Superiore di Sanità, Rome, Italy \\ **Dipartimento di Chimica Fisica ed Inorganica, Università di Bologna, \\ Viale del Risorgimento 4, 40136 Bologna, Italy
}

(Received June 5, 2000; Accepted September 5, 2000)

6-Methylcoumarin (I) is a synthetic fragrance material which has been largely used in the past in cosmetics and toiletries. ${ }^{1,2}$ It has been identified as a photocontact allergen both in humans and in guinea pigs; the mechanism of its photoallergenicity has been elucidated. ${ }^{2}$

3-Pyridine methanol (nicotynil alcohol) is an active principle which shows a vasodilator activity similar to that displayed by the corresponding acid; the form of hydrofluoride (II) is utilized in drugs and toothpastes due to its anticaries effect. ${ }^{3-5}$

The Italian law 713/86 (enclosure III) and subsequent adjournments, issued in compliance with an EEC Directive concerning cosmetic products, report I and II in the list of substances usable only for oral hygiene, and establish their maximum concentrations: $0.03 \%$ for $\mathrm{I}$ and $0.15 \%$, as hydrofluoride, for II.

In the literature, we have found only one paper dealing with the determination of $\mathrm{I}$ in fragrances and suntan products by HPLC, ${ }^{1}$ whereas, to our knowledge, there are no reported methods for the determination of II as free molecule in cosmetics and toiletries, but only methods describing the dosage of fluoride, which gives the salt with the pyridine nitrogen. ${ }^{6}$ Actually, two HPLC procedures have been developed for the routine analysis of II as one of the main metabolites of nicotine in biological fluids of smokers. ${ }^{7,8}$

Since cosmetics devoted to oral hygiene can contain both active principles, a simple analytical method that allows their simultaneous determination might be of some usefulness. This paper describes an HPLC assay suitable for the separation and determination of I, and II in cosmetic products, previously submitted to a very simple extraction.

\section{Experimental}

\section{Standards and reagents}

I and coumarin were purchased from Aldrich (Steinheim, Germany), II, in the form of hydrofluoride, was synthesized in our laboratory according to a patent by Fabre Pierre, S. A., while 3-pyridine methanol was supplied by BASF (Mannheim, Germany). Methyl-, ethyl-, and n-propyl-4-hydroxy benzoates (parabens) were purchased by Formenti (Milan, Italy). All reagents used were of analytical-reagent grade, and were used

$\dagger$ To whom correspondence should be addressed.

E-mail: tonelli@ms.fci.unibo.it without further purification. Acetonitrile and methanol were of HPLC grade. Water was deionized and doubly distilled from a glass apparatus. All solvents and solutions for HPLC analysis were filtered through a Millipore filter (pore size $0.45 \mu \mathrm{m}$ ) and vacuum degassed by sonication before use.

\section{Apparatus and HPLC conditions}

Chromatography was performed on a Shimadzu liquid chromatograph equipped with an external Rheodyne injection valve (Shimadzu, Kyoto, Japan), fitted with a $20-\mu \mathrm{L}$ sample loop, a Shimadzu SPD-M10 AV photodiode-array detector, and a personal computer to process the chromatographic data. The analytical column was of stainless-steel $(250 \mathrm{~mm} \times 4.0 \mathrm{~mm}$ i.d. $)$ packed with $5 \mu \mathrm{m}$ Purospher RP-18 (Merck, Darmstadt, Germany).

The initial mobile phase was a mixture of acetonitrile-water $(2: 98, \mathrm{v} / \mathrm{v})$, which was kept constant for $3 \mathrm{~min}$; a linear gradient elution was used to $60 \%$ acetonitrile in $15 \mathrm{~min}$; this composition was then maintained for additional $15 \mathrm{~min}$. At the end of elution, the initial mobile phase was passed through the column for $10 \mathrm{~min}$ to allow good re-equilibration of the chromatographic conditions. The flow-rate was $1.0 \mathrm{~mL} \mathrm{~min}{ }^{-1}$ and the detection wavelengths were 260 and $300 \mathrm{~nm}$ for II, and I, respectively. The range of wavelengths examined by the photodiode-array detector was $200-400 \mathrm{~nm}$. The temperature of the column was maintained at $25^{\circ} \mathrm{C}$.

\section{Calibration standard solutions}

A stock solution of I was prepared by dissolving about $20 \mathrm{mg}$ of the standard, exactly weighed, in $100.0 \mathrm{~mL}$ of methanol containing $0.25 \mathrm{mg}$ of coumarin as an internal standard (iS1). A set of working standard solutions was prepared by diluting aliquots of the stock solution, with methanol containing iS1, to give concentrations ranging from 0.2 to $10.0 \mu \mathrm{g} \mathrm{mL}-1$. A stock solution of II was prepared by dissolving about $30 \mathrm{mg}$ of the standard, accurately weighed, in $100.0 \mathrm{~mL}$ of methanol containing $3.0 \mathrm{mg}$ of methyl nicotinate as an internal standard (iS2). A set of working standard solutions was prepared by diluting aliquots of the stock solution, with methanol containing iS2, to give concentrations ranging from 0.2 to $100.0 \mu \mathrm{g} \mathrm{mL}^{-1}$.

The calibration graphs were constructed by plotting the ratio of the peak areas of I, and II to the relevant internal standard, obtained at the optimum wavelength of detection, versus the amounts (ng) injected. 


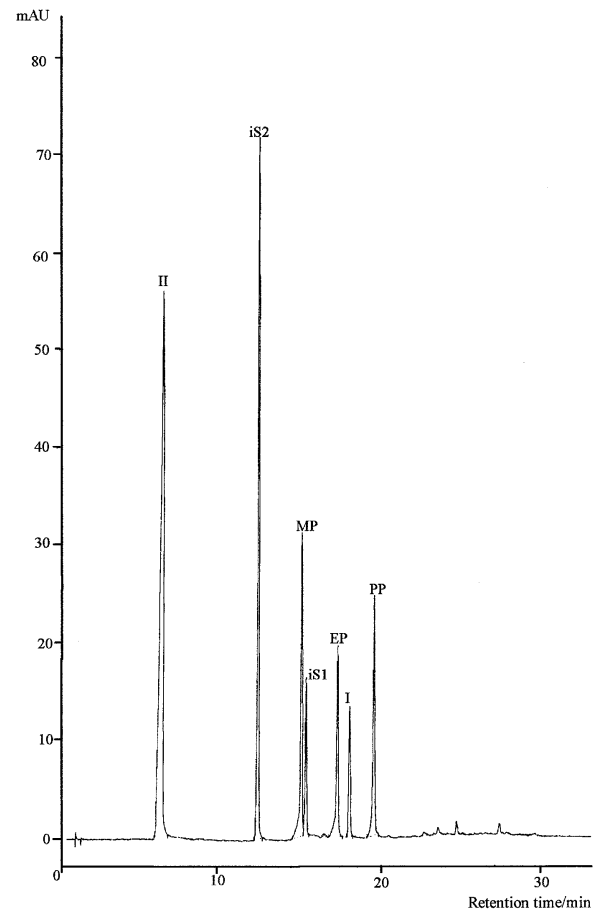

Fig. 1 Typical chromatogram obtained at $260 \mathrm{~nm}$ for solution A, containing $2.5 \mu \mathrm{g} \mathrm{mL}^{-1}$ and $30.0 \mu \mathrm{g} \mathrm{mL}^{-1}$ of I and II, respectively, and the preservatives studied.

\section{Sample preparation}

Samples of toothpastes and mouth-washes were prepared by the following procedure. About a 5-g amount of toothpaste was exactly weighed, added with $100.0 \mathrm{~mL}$ of methanol containing $3.0 \mathrm{mg}$ of methyl nicotinate and $0.25 \mathrm{mg}$ of coumarin (solution A) and submitted to an ultrasonic treatment for $10 \mathrm{~min}$. The resulting mixture was filtered through a $0.45 \mu \mathrm{m}$ filter and injected into the chromatograph. A $10.0-\mathrm{mL}$ volume of mouthwash was diluted with solution $\mathrm{A}$ and taken to volume in a $100.0 \mathrm{~mL}$ volumetric flask.

\section{Results and Discussion}

Figure 1 shows the typical chromatogram, recorded at $260 \mathrm{~nm}$, of a solution A containing the active principles I, II, at the concentrations indicated in the legend, and the preservatives methyl, ethyl, and $n$-propyl paraben (MP, EP, and PP). Since the cosmetic and toiletry matrices taken into account often contain preservatives, such as the esters of 4-hydroxybenzoic acid, which could interfere in the chromatographic separation, some of these active principles were analyzed together with the compounds of interest. In particular, the methyl, ethyl, and $n$ propyl parabens were considered.

The proposed stationary phase and the chosen elution conditions allowed a good separation with satisfactory symmetry of the peaks without appreciable tailing. Purosper RP-18 is a packing material which has been submitted to a multi-step chemical modification and deactivation of the surface in order to minimize silanol groups and heavy metals cations contents and, therefore, the possibility of complexes formation with basic compounds. The retention characteristics are summarized in Table 1. The retention times were reproducible under the experimental conditions used, the average coefficient of variation (C.V.) being less than $1.5 \%$.
Table 1 Chromatographic characteristics of tested compounds

\begin{tabular}{lrcc}
\hline Compound & $\begin{array}{c}\text { Retention time/ } \\
\text { min }\end{array}$ & $\begin{array}{c}\text { Detection wavelength/ } \\
\mathrm{nm}\end{array}$ & $\begin{array}{c}\text { Detection limit/ } \\
\text { ng injected }\end{array}$ \\
\hline II & 6.70 & 260 & 5.0 \\
i.S.2 & 12.31 & 260 & \\
MP & 15.05 & 260 & \\
i.S.1 & 15.33 & 300 & \\
EP & 17.31 & 260 & 2.5 \\
I & 18.10 & 300 & \\
PP & 19.56 & 260 & \\
\hline
\end{tabular}

Table 2 Analysis of toiletry products ${ }^{\mathrm{a}}$

\begin{tabular}{llll}
\hline \multicolumn{1}{c}{ Product } & \multicolumn{1}{c}{$(\mathrm{I})$} & \multicolumn{1}{c}{$(\mathrm{II})$} \\
& & \multicolumn{1}{c}{$(\%, \mathrm{w} / \mathrm{w})$} & \multicolumn{1}{c}{ n/w) } \\
\hline Mouth-wash & No. 1 & $0.020(0.002)$ & n.d. \\
& No. 2 & $0.020(0.001)$ & $0.036(0.002)$ \\
& No. 3 & n.d. & $0.030(0.002)$ \\
& No. 4 & $0.010(0.001)$ & $0.027(0.002)$ \\
& No. 5 & n.d. & $0.083(0.004)$ \\
Toothpaste & No. 1 & n.d. & $0.080(0.003)$ \\
& No. 2 & n.d. & $0.075(0.003)$ \\
& No. 3 & n.d. & $0.070(0.003)$ \\
No. 4 & n.d. & $0.065(0.003)$ \\
\hline No. 5 & 0.010(0.001) & \\
\hline
\end{tabular}

a. Mean of five determinations, S.D. in parenthesis. n.d. = not detected.

A detection wavelength of $260 \mathrm{~nm}$ was chosen, since this value permitted the simultaneous determination of all of the compounds investigated with good sensitivity. If the chromatographic run is performed while setting the detector at $300 \mathrm{~nm}$, only the peaks relevant to I and coumarin are observable, since the other compounds investigated display very low molar absorptivities at this wavelength. Therefore, if only I is to be determined, the detection can be performed at $300 \mathrm{~nm}$ to minimize interferences from other active principles or excipients present into the toiletry sample. The photodiodearray detector allowed an estimation of the peak purity factors. These values were calculated over the range $200-400 \mathrm{~nm}$, and resulted in $>99.5 \%$ for all of the agents studied, thus confirming the good resolution achieved.

The calibration graphs were constructed from five consecutive injections over the covered ranges of concentration, as indicated in the experimental section. A least-squares regression fit showed good linearity, passing through the origin. The equations obtained for the calibration lines for I and II, respectively, were $y=-0.017( \pm 0.05)+1.434(0.006) x\left[R^{2}=\right.$ $0.9993]$ and $y=-0.024( \pm 0.07)+0.616(0.005) x\left[R^{2}=0.9996\right]$, where $y$ represents the peak area and $x$, the sample size. The detection limits, calculated as the signal-to-noise ratio of 3 , were 2.5 and $5.0 \mathrm{ng}$ injected for II, and I, respectively, at the optimum detection wavelengths.

The content of the agents under study in 10 commercially available cosmetic formulations was determined in triplicate by using the proposed method. Some samples contained I and II in combination, whose identity was confirmed by comparing the retention times and the purity factor values of the peaks observed with those obtained from the standard solutions. The assay results are given in Table 2. Typical chromatograms obtained for a sample of toothpaste and a mouth-wash, 


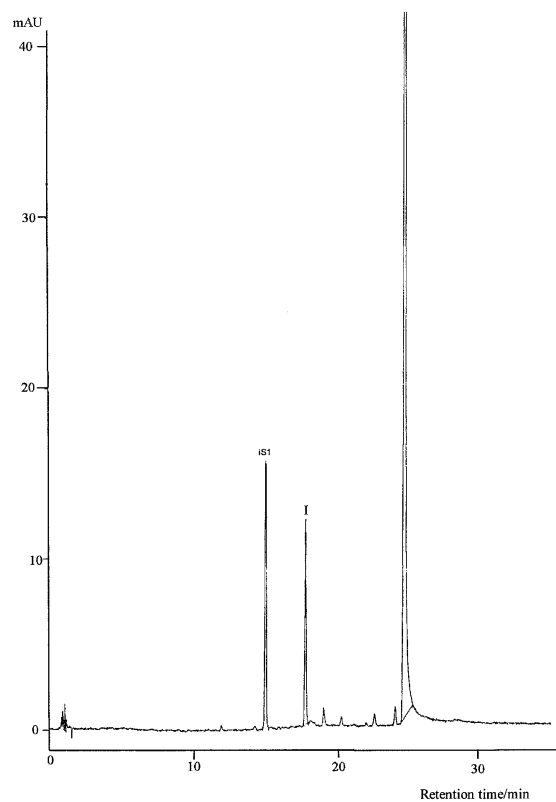

Fig. 2 Chromatogram obtained at $260 \mathrm{~nm}$ for a sample of toothpaste No. 2 (see Table 2).

respectively, are shown in Figs. 2 and 3. From Fig. 2, it is evident that the sample does not contain I. The big peak centred at $15.10 \mathrm{~min}$ corresponds to MP, as confirmed by the value of the purity factor. In the chromatogram reported in Fig. 3, which was obtained by injecting a sample of mouth-wash No. 4, the peak relevant to II does not appear, since it has been recorded at $300 \mathrm{~nm}$. In conclusion, the analytical results obtained demonstrate that the developed HPLC procedure, together with simple sample processing, can be a valuable tool for quality control studies, especially to verify the compliance of toiletry products for oral hygiene with current legislation.

\section{References}

1. A. Bettero and C. A. Benassi, J. Pharm. Biomed. Anal., 1983, $1,229$.

2. S. Kato, T. Seki, Y. Katsumura, T. Kobayashi, K. Komatsu,

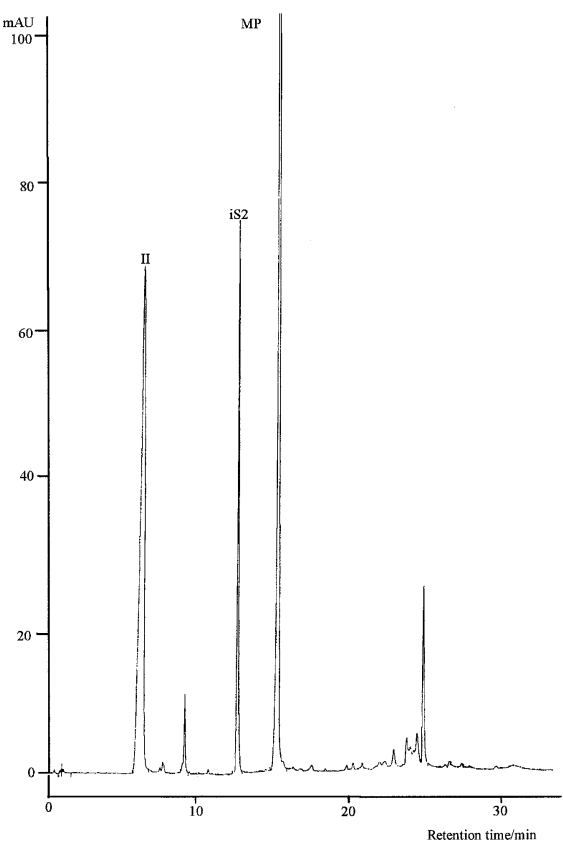

Fig. 3 Chromatogram obtained at $300 \mathrm{~nm}$ for a sample of mouthwash No. 4 (see Table 2).

and S. Fukushima, Toxicol. Appl. Pharmacol., 1985, 81, 295.

3. J. C. Vezin, G. M. Bounoure, J. Mondain, and P. Poitou, Pharm. Acta Helv., 1985, 60, 145.

4. E. Tadmor, P. Poitou, and I. Gedalia, J. Oral Rehabil., 1989, 16,613 .

5. H. Lauressergues and J. Mondain, Ther. Pharmacol. Clin., 1985, 4,3 .

6. C. Pham Huy, F. Nadji, M. Postaire, and M. Hamon, Ann. Pharm. Fr., 1991, 49, 39.

7. M. T. Parviainen, E. V. J. Puhakainen, R. Laatikainen, K. Savolainen, J. Herranen, and R. D. Barlow, J. Chromatogr., 1990, 525, 193

8. P. P. Rop, F. Grimaldi, C. Oddoze, and A. Viala, J. Chromatogr., 1993, 612, 302.

9. H. Cousse, G. Mouzin, J. C. Vezin, J. Dubois, and L. D. D'Hinterland, Ger. Offen. 2,633,028, 10 Feb 1977, Fr. Appl. 75/23, 715; Chem. Abstr., 1977, 86, 189710 w. 